\title{
Development and Experimental Validation of a Dynamic Model for a Fresnel Solar Collector ${ }^{\star}$
}

\author{
María Robledo* Juan M. Escaño** Amparo Núñez ${ }^{* * *}$ \\ Carlos Bordons ${ }^{* * *}$ Eduardo F. Camacho ${ }^{\dagger}$
}

University of Seville. Escuela Superior de Ingenieros. Dto. Ingeniería de Sistemas y Automática. Avda. de los Descubrimientos s/n

\author{
*marrobvaz@alum.us.es \\ **jmesca@cartuja.us.es \\ ***amparo@cartuja.us.es \\ ****bordons@cartuja.us.es \\ $\dagger$ †eduardo@cartuja.us.es
}

\begin{abstract}
Fresnel collector field of a solar refrigeration plant. The plant is located in the Escuela Superior de Ingenieros of the University of Seville. The dynamic model parameter model developed can be used as a control model or as a simulation tool to test controllers. The lumped parameters have been determined by using real data from the plant in different operating conditions. The model has been validated against a data validation set obtained from the plant. The model has shown to reproduce the system behavior with a good compromise in accuracy and model complexity.
\end{abstract}

Keywords: Modelling, Solar Plant

\section{INTRODUCTION}

Undoubtedly, solar energy is one of the most important renewable energy there. This year the sun shed 4000 times more energy than they consume, so their potential is almost unlimited.

Spain and Andalusia in particular, for its privileged location and climate, is particularly favored compared to other European countries, as on every square meter of land per year affecting about 1500 kilowatt-hours of energy. This energy can be used directly or be converted into other useful forms, for example, electricity.

It would be irrational not to try to take advantage, by all means technically possible, this free energy source, clean and renewable, which can free us from dependence on oil or other unsafe alternatives, pollutants or simply exhaustible. In recent years there has been growth substantial cooling requirements in buildings, service sector, both requirement for greater comfort conditions as increased thermal load due to the extensive use of personal computers and increased use of lighting.

However, there are technologies for cooling, driven by heat sources such as solar energy. When using a solar cooling system in addition to inherent advantages of using a renewable energy source, it should be noted the coincidence between peak demand and peak production (solar maximum). The solar energy use for cooling, can be done by using solar collectors that produce heat to medium or high temperature.

The Fresnel solar concentrator systems are a new technol-

* This work was supported by the Spanish National project DPI2008-05818 and the Andalusian project P07-TEP-02720. ogy that aims to make it in direct competition with the cylinder-parabolic type. They are systems of linear focus, ie focus solar radiation along a line that corresponds to a solar absorption tube for circulating a thermal fluid.

The advantages of using Fresnel solar collectors compared to parabolic, are:

- Mirrors and tracking system at low cost.

- Fixed absorber tube, no need for high pressure flexible joints.

- Greater tolerance to extreme temperatures.

- No need for vacuum tubes.

- No need for expansion elbows (the absorber is free at one end to dilate).

- Reflectors flat located close to the ground, reduced wind loads.

- Efficient use of land, the ranks of collectors can be placed close to each other.

- Easy access to moving parts and superficial.

It is estimated that the cost reduction due to the use of Fresnel technology compared to cylinder-parabolic collectors, will be around 50\% when the technology has developed enough (Häberle et al. 2006)

For thermal applications at the building, currently under way some developments, such as the German company PSE GmbH (Camacho et Al. 1992), which is testing a small prototype in headquarters of Freiburg (Germany) and another in conjunction with equipment manufacturer Robur absorption in Bergamo (Italy), in addition to the facility located in the School of Industrial Engineering at Sevilla and is the subject of this article. 


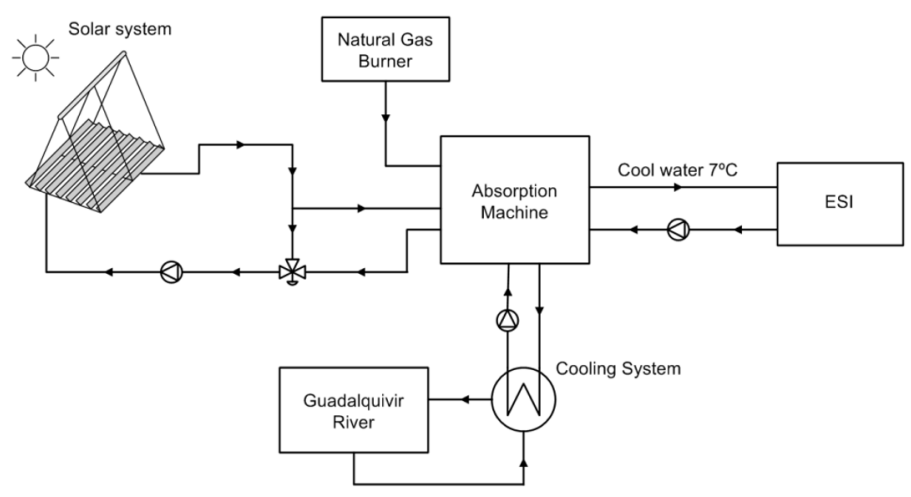

Fig. 1. Plant Scheme

This paper is organized as follows: Section 2 presents a description of the real plant located in Seville. Section 3 shows the operational models. Section 4 presents the dynamical model. In section 5 validation graphics are presented and in section 6 presents the conclusions.

\section{PLANT DESCRIPTION}

Solar cooling plant is located on the roof of the Escuela Superior de Ingenieros, University of Sevilla. The sun's radiation is collected as heat in a solar collector concentration Fresnel, using water as heat transfer fluid at $180^{\circ} \mathrm{C}$ and 1.3 MPa. The superheated water is used to power air conditioning machine of double-effect absorption of 174 $\mathrm{kW}$, which transforms the thermal energy in cold climate suitable for various departments of the School. When irradiation is insufficient to power the absorption chiller, the energy supplied by natural gas, provides support to keep the process running. Figure 1 shows an overview of the plant, where you can see the main components of the same: solar system, auxiliary power system and cooling system.

\subsection{Process Heat Collector}

The solar system consists of a set of solar collectors type Fresnell (Häberle et al. 2006). These are systems of linear focus, ie they concentrate solar radiation along a line that corresponds to a absorbtion tube through which circulates a heat transfer fluid (in our case, water).

The hub system is made up of reflective surfaces with high curvature radius of intercepting and directing reflected solar radiation to the receiver tube located on a different plane of reflection. As the aim is to concentrate sunlight on the surface of the receiver, the concentrator system must have a control mechanism that allows you to track the sun, so that is always focused on it.

Once the concentrated radiation reaches the receiver, it converts into heat energy by transferring energy to the working fluid. The elements that make up this new configuration of collector are:

- Primary mirrors (reflectors concentrating solar radiation levels), made of glass security, slightly curved elastically (with a radius of curvature between 8.6 and $10.6 \mathrm{~m}$ ) and attached to the support structure, which is driven by a drive mechanism. This mechanism is part of the tracking system and operates independently. Each drive mechanism moves eight mirrors in a row, four on each side. In total we have 11 rows getting reflective surface $352 \mathrm{~m} 2$.

- Receiver, consisting of a tube or more absorber tubes, with or without cover. The receiver is in a plane parallel and superior to the concentrators planes. From inside the absorber tubes carrying fluid circulates. The absorber tube is surrounded by a glass cover to ensure the vacuum between them.

- Secondary reflector, consisting of a thin metal casing and a mirror (reflectivity rating: 0.77) mounted inside the cap. Its mission is to reflect solar radiation has no direct impact on the receiver from the primary mirror, thus optimize the optical efficiency of the system. It also protects the receiver tube.

- Supporting structure of the whole. The solar collector is connected to the rest of the hydraulic circuit. The temperature in the hydraulic circuit is limited to a maximum of $200^{\circ} \mathrm{C}$ and a maximum pressure of 16 bar. The standard operating pressure is limited to 13 bar, and pressure relief valve is set to 16 bar. Nominal water flow is $13 \frac{\mathrm{m}^{3}}{\mathrm{~h}}$.

\subsection{Auxiliary Power System}

The auxiliary power system consists of a natural gas boiler with enough power, which is responsible for supplying the energy needed when solar radiation is insufficient

\subsection{Absorption machine}

It transforms the thermal energy (hot water) in cold water to be used by the building's HVAC equipment. It is based on technology transfer thermal energy from a cold to hot by evaporating low pressure refrigerant fluid. In this case, instead of using a compressor, used as a source of heat, water at $180^{\circ} \mathrm{C}$ from the solar collector and boiler associated with the absorption machine, obtaining the necessary power for operation. Is an absorption machine, double effect cycle with $\mathrm{LiBr} /$ water. It has a cooling power of $174 \mathrm{kK}$ and a cold theoretical COP of 1.34. The hot water temperature input is $165^{\circ} \mathrm{C} / 180^{\circ} \mathrm{C}$. A cooling fluid is necessary in the absorption machine, besides the hot thermal fluid. This refrigerant is obtained from water catchment of the river Guadalquivir. Circuit that was created for the buildings of the 1992 Universal Exhibition.

In the case of this plant, we have a variant with respect to a standard solar cooling plant: the heat transfer fluid is used directly to drive the absorption chiller. In this way only requires a solar water circuit, avoiding the need for downstream separation equipment circuits and heat transfer (heat exchangers, steam generators, etc.) and simplifying the hydraulic system of the solar side of the installation.

\subsection{Control System}

The control system will optimize the system performance by acting on the valves, starting and stopping the solar 


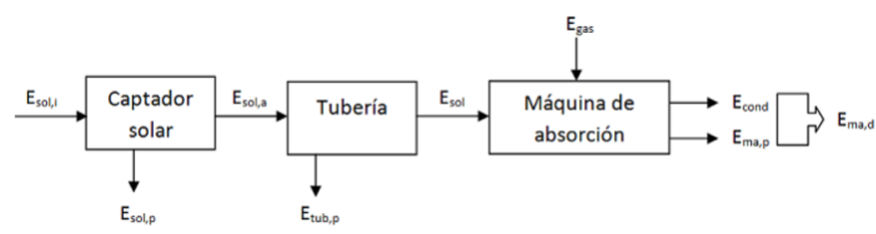

Fig. 2. Operating diagram

collector, the absorption machine and hydraulic pumps and reporting of possible breakdowns or incidents. Serves also to extract information about the flow, consumption and temperatures in different parts of the system, data to be used in research work. The system can be operated through network, using a webserver included in the main control module.

\section{OPERATING MODES}

The energy supply for the operation of the absorption chiller can come from solar energy incident on the Fresnel field and/or energy provided by natural gas. Figure 2 shows the diagram of operation of the plant. Where Esol, $i$ is the solar energy incident on the solar collector, Esol,a, the energy absorbed, Esol, $p$ and Etub, $p$ are lost in the solar collector and piping circuit, Esol the energy given to the absorption machine, Egasa the energy given by combustion of natural gas and Ema,d is the energy dissipated from the absorption chiller as the sum of the condensation Econd and smoke and unburned carbon losses Ema,p.

The absorption machine has two distinct operating modes:

- Only gas: The heat introduced into the generator comes from the combustion of natural gas in the burner, Egen = Egas.

- Mixed: The heat introduced into the generator comes from combustion of natural gas and water flow at high temperature from the Fresnel solar collector, Egen = Egas + Esol. To optimize the performance of mixed mode, water from the solar collector should be $180^{\circ} \mathrm{C}$. Añadir gráficas

\section{DYNAMIC MODEL OF THE FRESNEL COLLECTOR}

There is a previous thermal model to study the efficiency of this plant and for identifying design improvements (Bermejo et al. 2010 ). It is important to have a model to describe the main dynamics of the collector in order to simulate a diversity of operating conditions, disturbances. For that purpose, a concentrated parameter model has been developed in this work.

\subsection{Concentrated parameter model}

This section is devoted to describing the procedure for obtaining a concentrated parameter model of the solar collector plant. The variation in the internal energy of the collector can be described (Camacho et al. 1997 ) by:

$$
C \frac{d T}{d t}=n_{0} S I-\dot{q} P_{c p}\left(T-T_{i n}\right)-H_{l}\left(T_{m}-T_{a}\right)
$$

Where $T$ is the outlet temperature on the collector, $I$ is the effective solar radiation, $T_{i n}$ is the inlet temperature, $T_{a}$ is the ambient temperature, $T_{m}$ is the mean inletoutlet temperature, $\dot{q}$ is the water flow, $n_{0}$ is the optical efficiency, $S$ is the effective surface, $C$ is the specific thermal capacity of the fluid, $P_{c p}$ is a term that accounts for the product and quotient of characteristic magnitudes (areas, thermal capacities, etc.) and $H_{l}$ is the global thermal losses coefficient.

Due to the variation of $H_{l}$ with the temperature, we will consider that:

- $H_{l}\left(T_{m}-T_{a}\right)=h_{l 1}\left(T_{m}-T_{a}\right)+h_{l 2}\left(T_{m}-T_{a}\right)^{2}$ and

- $P_{c p}\left(T-T_{i n}\right)=p_{0}\left(T-T_{i n}\right)+p 1\left(T-T_{i n}\right)^{2}$

Then,

$$
\begin{aligned}
C \frac{d T}{d t}= & n_{0} S I-\dot{q}\left[p_{0}\left(T-T_{i n}\right)+p 1\left(T-T_{i n}\right)^{2}\right] \\
& -h_{l 1}\left(T_{m}-T_{a}\right)+h_{l 2}\left(T_{m}-T_{a}\right)^{2}=C f(t)
\end{aligned}
$$

and

$$
T(t+\Delta t)=T(t)+\Delta t \cdot f(t)
$$

Data for the model are obtained from the monitoring system. From it can be collected the values of the variables $T_{a}, T_{i n}, \dot{q}, T, I_{d}$, being $I_{d}$ the direct solar radiation.

\subsection{Solar radiation}

The direct solar radiation is not effective, which is what is needed. To calculate, you have to take into account a number of factors to calculate:

- The shadows between the mirrors themselves.

- The optical losses by not affect the sun's rays perpendicularly on the tube.

- The part of the tube which is not illuminated by mirrors

Under the guidance of the plant, its location and the date and time (from there you can calculate the inclination and azimuth) can calculate losses.

First of all, we will define the reference system and its position with respect to the geographic axis.

The solar field is located parallel to the south facade of Engineering School of Seville, with a deviation of $12^{\circ} 3$ ' 11 " west. Each row measures 0.5 meters wide and are separated from each other by a distance of 0.2 meters. The absorber tube is located 4 meters above the mirrors. The coordinate origin was located in the center of the row number 6 (middle row).

To calculate the solar incidence angle in the mirror, we need to know the azimuth $(A z i)$ and solar elevation(Ele), which depend on the day and time. As the mirrors have a tracking system along the $\mathrm{Z}$ axis, in order to vary its inclination according to the solar elevation is projected solar vector incidence in the axes $\mathrm{X}$ and $\mathrm{Y}$ to calculate the slope of the same.

In this way we obtain a vector of incidence in two dimensions with the following expression:

$$
\vec{\imath}_{2 D}=\left[\begin{array}{c}
\cos (\text { Ele }) \cdot \cos (- \text { Azi }+ \text { Orientation }) \\
\sin (\text { Ele })
\end{array}\right]
$$




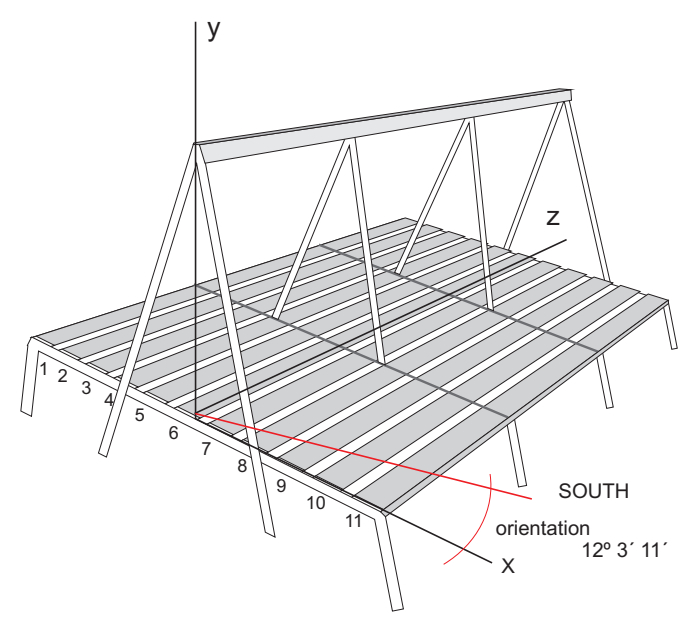

Fig. 3. Mirrors system

Where Orientation is the angle showed in figure 3

Having calculated the vector of incidence, it is imperative that the vector with which solar radiation is reflected in the mirrors take direction from the mirror to the absorber tube. Using the calculation for the midpoint of each row will mirror position $\left(X_{E}, Y_{E}\right)$ with $Y_{E}=0$, we have the following table:

\begin{tabular}{|c|c|}
\hline FILA & $X_{E}$ (meters) \\
\hline 1 & -3.5 \\
2 & -2.8 \\
3 & -2.1 \\
4 & -1.4 \\
5 & -0.7 \\
6 & 0.0 \\
7 & 0.7 \\
8 & 1.4 \\
9 & 2.1 \\
10 & 2.8 \\
11 & 3.5 \\
\hline
\end{tabular}

Knowing this and the coordinate of the absorber tube (0.4) one can calculate the vector of reflection of solar radiation.

$$
\vec{r}_{2 D}=\left[\begin{array}{l}
r_{2 D x} \\
r_{s D y}
\end{array}\right]=\left[\begin{array}{c}
\frac{X_{t}-X_{E}}{\sqrt{\left(X_{t}-X_{E}\right)^{2}+\left(Y_{t}-Y_{E}\right)^{2}}} \\
\frac{Y_{t}-Y_{E}}{\sqrt{\left(X_{t}-X_{E}\right)^{2}+\left(Y_{t}-Y_{E}\right)^{2}}}
\end{array}\right]
$$

The angle of reflection vector to the horizontal is $\beta$. The angle between the vector of incidence with the reflection vector, $\alpha$, is given by the scalar product.

$\vec{\imath}_{2 D} \cdot \vec{r}_{2 D}=\left|\vec{\imath}_{2 D}\right| \cdot\left|\vec{r}_{2 D}\right| \cdot \cos \alpha$

$\alpha=\arccos \left(\frac{\vec{\imath}_{2 D} \cdot \vec{r}_{2 D}}{\left|\vec{\imath}_{2 D}\right| \cdot\left|\vec{r}_{2 D}\right|}\right)$ and $\beta=\frac{\alpha}{2}$

To calculate the inclination of the mirrors are going to distinguish three possible cases:

- $X_{E}<0$ (rows 1 to 5 ). See figure $4 \gamma \geq \beta \rightarrow$ incl $=\frac{\pi}{2}-$ $\beta-\theta$ (a) $\gamma<\beta \rightarrow$ incl $=\frac{\pi}{2}-\beta+\theta$ (b)

- $X_{E}=0$, inclin $=\theta$ In this case, the vector of reflection is perpendicular to the horizontal because the tube absorber is just above the center of the mirror (c)

- $X_{E}>0$ (rows 7 to 11 ). (d) incl $=\beta+\theta-\frac{\pi}{2}$
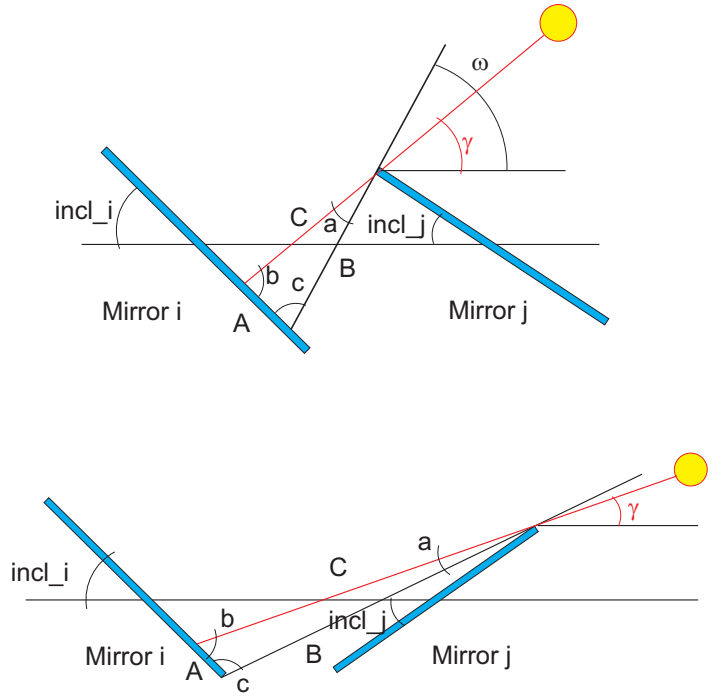

Fig. 5. shaded areas

After calculating the inclination of the rows we can begin to calculate the different shadows produced on the collector Fresnell:

- Self-shadowing between mirrors. To calculate the shadows they cast the mirrors each other is also going to use the above model in two dimensions. To calculate the shaded areas in each row of mirrors is to resort to simple geometry. Once we know this area will be divided by the total area to obtain a shading factor for each row. To calculate these shadows will consider several cases:

Case 1 Row with positive inclination, and the next with positive inclination. (see figure 5-a).

Case 2 Row with positive inclination, and the next with negative inclination. (see figure 5-b). The shaded portion is calculated the same way as the previous case.

Case 3 Row with negative inclination, and the next with negative inclination. In this case there is no shadow

It has been estimated length of shadow in each row. To calculate the shaded area of each row, we multiply by 4 (the length of the mirror) and 16 (there are 16 mirrors in each row). To calculate the factor of the shadow of each row must be divided by $32 \mathrm{~m}^{2}$ (total area of each row of mirrors). $f_{\text {shadow }}=A \cdot 4 \cdot 32$

- Optical Loss Factor. Total incident radiation could be exploited fully, if it were to mirrors in a direction perpendicular to them. This does not happen because the mirrors are positioned so that reflected radiation go to the absorber tube, so that shows a loss. These losses are quantified by the angle $\delta$ (angle of the mirror with the normal vector of incidence). There are two possible cases:

(1) $\delta=\frac{\pi}{2}-\gamma-$ incl with incl $<0$

(2) $\delta=\pi-\left(\frac{\pi}{2}-i n c l\right)-\gamma=\frac{\pi}{2}+i n c l-\gamma$ with incl $>0$ The optical loss factor is $\cos \delta$

- Unlit section of the tube. To calculate the length of tube absorber that is shaded, we need a threedimensional model since it has to take into account the solar azimuthal component. You need the normal 
vector at each mirror. The $\mathrm{z}$-axis varies from 0 to $64 \mathrm{~m}$ (the length of the absorber tube.)

$$
\vec{n}=\left[\begin{array}{c}
\sin (i n c l) \\
\cos (i n c l) \\
0
\end{array}\right]
$$

$\vec{\imath} \times \vec{n}=\vec{n} \times \vec{r}_{3 D}$ The reflection vector in $3 \mathrm{D}$ is:

$$
\vec{r}_{3 D}=\left[\begin{array}{c}
\frac{X_{t}-X_{E}}{\sqrt{\left(X_{t}-X_{E}\right)^{2}+\left(Y_{t}-Y_{E}\right)^{2}+\left(Z_{t}-Z_{E}\right)^{2}}} \\
\frac{Y_{t}-Y_{E}}{\sqrt{\left(X_{t}-X_{E}\right)^{2}+\left(Y_{t}-Y_{E}\right)^{2}+\left(Z_{t}-Z_{E}\right)^{2}}} \\
\frac{Z_{t}-Z_{E}}{\sqrt{\left(X_{t}-X_{E}\right)^{2}+\left(Y_{t}-Y_{E}\right)^{2}+\left(Z_{t}-Z_{E}\right)^{2}}}
\end{array}\right]
$$

Getting $Z_{t}$, from the previous equations, the unlit section is obtained:

(1) if the shadow is caused by the east side: unlit $_{\text {row }}=$ Total_length $-Z_{t}$

(2) if the shadow is caused by the west side: unlit $_{\text {row }}=Z_{t}$

Once we know the length of the unlit tube which causes each row, calculate the correction factor: $f_{\text {unlit }}$ ow $=\frac{\text { Total_length-unlit } \text { row }_{\text {-len }}}{\text { Total_length }}$

And finally, we can calculate the total correction factor, which covers all losses: $f_{\text {loss }_{i}}=f_{\text {unlit }_{i}} \cdot \cos \delta_{i} \cdot f_{\text {shadow }}$

The total radiation received by the absorber tube can be easily calculated as follows:

$$
I_{T}=\sum_{i=1}^{11} I_{d} \cdot A_{i} \cdot f_{\text {loss }_{i}}
$$

Where $A_{i}$ is the area of each mirrors row.

Using least mean squares method to obtain the parameters in equation 4.1, and experimental setting after that, the model is:

$$
\begin{array}{r}
\frac{d T}{d t}=7.961 \cdot 10^{-7} I_{T}-0.150 \dot{q}\left(T-T_{i n}\right) \\
-6.262 \cdot 10^{-6} \dot{q}\left(T-T_{i n}\right)^{2}-1.179 \cdot 10^{-5}\left(T_{m}-T_{a}\right) \\
+12.606 \cdot 10^{-4}\left(T_{m}-T_{a}\right)^{2}
\end{array}
$$

\section{VALIDATION OF THE MODEL}

To validate the model there had been used data set of several days. Figure 6 shows the behavior of the model to real data four days.

\section{CONCLUSIONS}

The paper has presented a lumped parameter dynamic model of a Fresnel collector field. The model main objective is to serve as a control model for model based controller or for testing controllers behavior under simulation. The model parameters have been obtained using data from the real plant. The model has been validated by comparing the model variables with data obtained from the plant. The mode has shown to reproduce the plant behavior with enough accuracy in spite of its simplicity.

\section{REFERENCES}

[Häberle et al. 2006] Häberle A, Berger M, Luginsland F, et al. "Linear concentrating Fresnel collector for process heat applications". Proceedings of the 13th International Symposium on Concentrated Solar Power and Chemical Energy Technologies: Spain. (2006).

[Camacho et Al. 1992] E.F. Camacho, Rubio an Hughes, "Selftuning control of a solar power plant with a distributed collector field", IEEE Control Systems Magazin, pp. 72-78, April 92.

[Camacho et al. 1997 ] E.F. Camacho, M. Berenguel, F.R. Rubio. Advanced Control of Solar Plant. Springer, London, 1997.

[Bermejo et al. 2010 ] P. Bermejo, F. J. Pino, F. Rosa. Solar absorption cooling plant in Seville. Solar Energy 84 (2010) 15031512

[Saying and McVeigh 1992 ] A. M. Sayigh and J.C. McVeigh (editors)."Solar Air Conditioning and Refrigeration". Pergamon Press, 1992.

[García 2006] X.García Casals, "Solar absorption cooling in Spain: Perspectives and outcomes from the simulation of recent installations", Renewable Energy,2006, Vo 31, pages=1371-1389,

[Balaras et Al. 2007] A.C. Balaras and G. Grossman and H.M. Henning and C. A.I. Ferreira and E. Podesser and L. Wang and E. Wiemken, "Solar air conditioning in European overview", Renewable and Sustainable Energy Reviews, Vo 11, pages $=299-314,2007$

[Silva et Al. 2002] R. N. Silva, L. M. Rato and J.M. Lemos. "Observer based non uniform sampling predictive controller for a solar plant", 15th IFAC World Congress, Barcelona 2002.

[Nuñez and Bordons. 2005] A. Núñez-Reyes, J.E. Normey-Rico and C. Bordons and E.F. Camacho," A Smith predictive based MPC in a solar air conditioning plant",Journal of Process control, 2005 , Vo $15 / 1$, pages $=1-10$,

[Nuñez and Bordons. 2005] A.Núñez-Reyes and C. Bordons, "Predictive control of a solar air conditioning plant with simultaneous identification",44th IEEE Conference on Decision and Control and European Control Conference CDC-ECC'05, 2005, Sevilla, España

[Nuñez et Al. 2002] A.Núñez-Reyes and C. Scheffer-Dutra and C. Bordons, "Controle preditivo com restrições aplicado a uma planta solar de climatização",XIV Congresso Brasileiro de Automatica. CBA'02, 2002, Natal, Brasil 

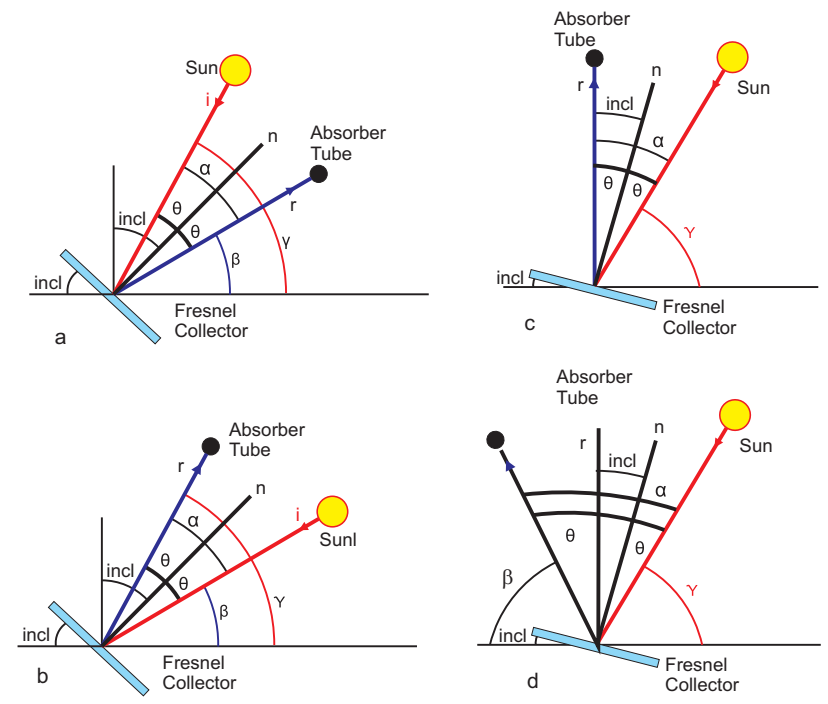

Fig. 4. Inclination angle
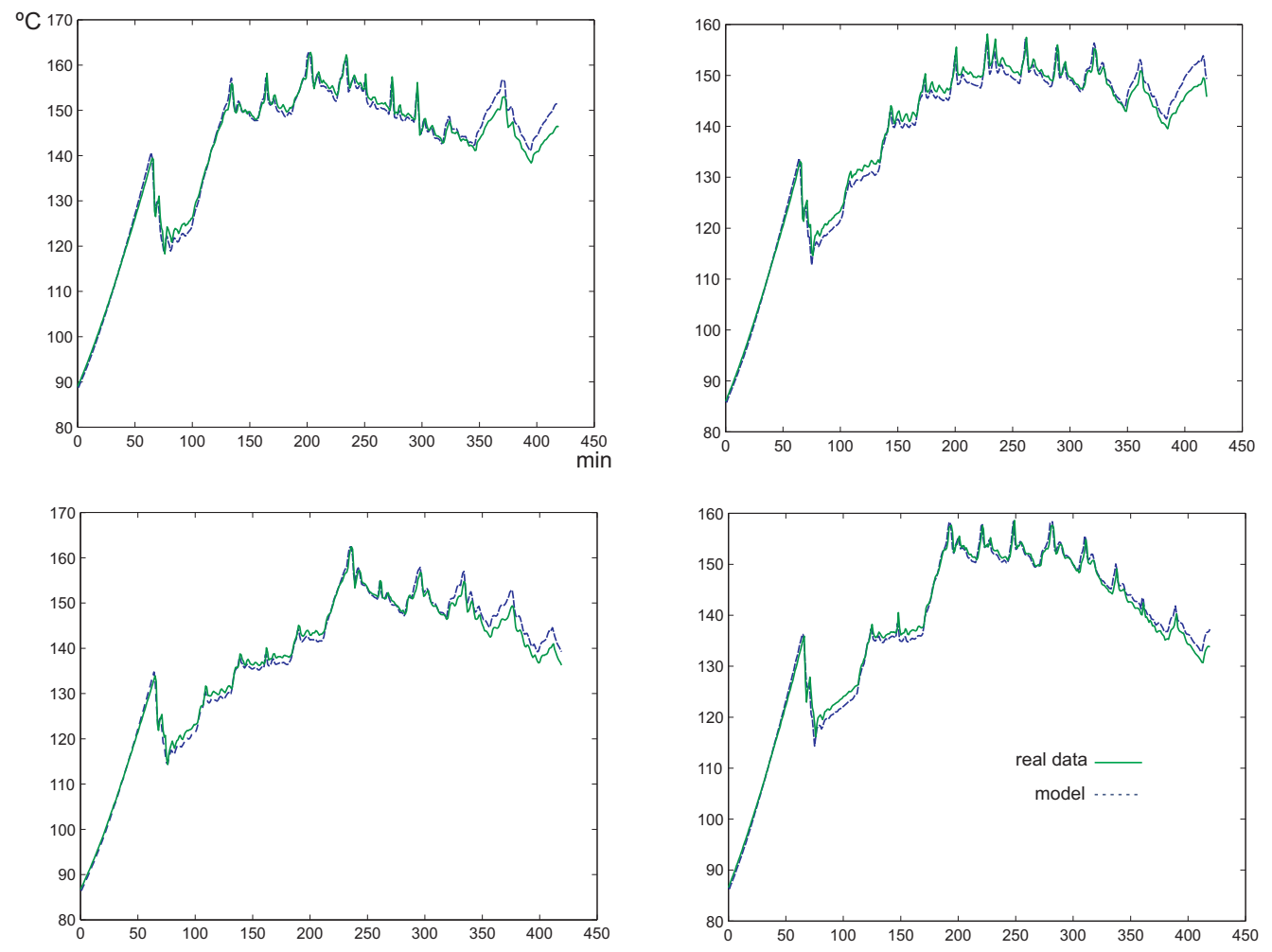

Fig. 6. validation of the concentrated parameters model 